\title{
Article \\ Impact of the Result of Soccer Matches on the Heart Rate Variability of Women Soccer Players
}

\author{
Rosa $\mathrm{M}^{\mathrm{a}}$. Ayuso-Moreno ${ }^{1}$, Juan Pedro Fuentes-García ${ }^{1, *(\mathbb{D},}$, Hadi Nobari ${ }^{2,3}{ }^{(\mathbb{D}}$ and Santos Villafaina ${ }^{4}$ (D) \\ 1 Faculty of Sport Science, University of Extremadura, Avda. Universidad S/N, 10003 Cáceres, Spain; \\ roayusom@alumnos.unex.es \\ 2 Sports Scientist, Sepahan Football Club, Isfahan 81887-78473, Iran; hadi.nobari1@gmail.com \\ 3 HEME Research Group, Faculty of Sport Sciences, University of Extremadura, 10003 Cáceres, Spain \\ 4 Physical Activity and Quality of Life Research Group (AFYCAV), University of Extremadura, 10003 Cáceres, \\ Spain; svillafaina@unex.es \\ * Correspondence: jpfuent@unex.es
}

Citation: Ayuso-Moreno, R.M Fuentes-García, J.P.; Nobari, H.; Villafaina, S. Impact of the Result of Soccer Matches on the Heart Rate Variability of Women Soccer Players. Int. J. Environ. Res. Public Health 2021, 18, 9414. https://doi.org/10.3390/ ijerph18179414

Academic Editor: Paul B. Tchounwou

Received: 17 August 2021

Accepted: 5 September 2021

Published: 6 September 2021

Publisher's Note: MDPI stays neutral with regard to jurisdictional claims in published maps and institutional affiliations.

Copyright: (c) 2021 by the authors. Licensee MDPI, Basel, Switzerland This article is an open access article distributed under the terms and conditions of the Creative Commons Attribution (CC BY) license (https:// creativecommons.org/licenses/by/ $4.0 /)$

\begin{abstract}
The present study aimed to evaluate the effects of a match lost and a match won on post-competitive heart rate variability (HRV) in semi-professional female soccer athletes. A total of 13 players, with a mean age of 23.75 (5.32), from the Cáceres Women Football Club of the Spanish Second National Division participated in our study. They were evaluated in two microcycles which correspond to a match lost and a match won. For each microcycle, baseline and post-competitive measures were collected. Results indicate that HRV was significantly reduced before a match lost and won. Significant differences in HRV variables were observed when compared the lost match, and the match won. Results highlight the importance and usefulness of analyzing the HRV as an indicator of post-competitive fatigue in semiprofessional soccer players. Therefore, a competition's results could be considered a relevant variable to consider when programming training load.
\end{abstract}

Keywords: female; football; autonomic modulation; fatigue; training load

\section{Introduction}

Biological signals are used as tools for controlling and evaluating training loads and acute and chronic effects on the athlete's body [1,2]. According to the scientific literature and as technology advances, there is growing interest in monitoring training loads in athletes to control their adaptation [3]. In team sports, monitoring training load is crucial for optimizing performance and preventing injuries, enabling us to anticipate the emergence of overtraining $[4,5]$. Thus, biomarkers that provide us information regarding changes in athlete's fatigue are highly appreciated.

Heart rate variability (HRV) is a noninvasive index that evaluates the balance between sympathetic and parasympathetic activity due to the study of successive heartbeats variation over an interval of time [6]. A reduced HRV, induced by a predomination of sympathetic activity, has been related to a reduced regulatory capacity to adapt to different challenges such as exercise or cognitive stressors [7]. Furthermore, a decrease in HRV has been considered a marker of fatigue, poor cardiovascular adaptation to effort, and overtraining [8,9], and, consequently, it has also been correlated with low sports performance [10].

Technological advances have made it possible to improve the fatigue management of athletes through the analysis of HRV [3,9]. HRV has been used in team sports as a sport-specific indicator based on data extracted from training and competitions [11]. In this regard, the study of HRV in team sports is crucial for optimizing performance and preventing injuries, fatigue, or overtraining [12]. In soccer, previous studies have used the HRV to control and manage training load during seasons [13-18]. However, in female soccer players, studies which analyze HRV are limited. In this regard, previous studies used the HRV to control training load [19], fatigue [20], or precompetitive anxiety [21]. 
Fatigue induced by sport is a usual situation within training and competition, but if it is not controlled, it can lead to negative alterations [22]. It is a process that has an effect on some variables of physical performance (technique or precision), and that must be taken into account in football training and recovery [23-25]. In this regard, a previous study showed that depending on the quality of the teams against which one competes, the distance and the intensity are different, which influences the players' fatigue [26]. Furthermore, HRV is also sensitive to cognitive processes [27,28], such as emotions [29]. In this regard, previous studies have reported that athletes experienced mood and wellbeing changes after a loss $[30,31]$. Specifically, athletes reported higher depression, anxiety, social dysfunction, and anger after a loss, compared to a win, while lower levels of vigor were observed after a loss, compared to after a win [31]. Furthermore, previous studies have found that soccer players who lost a match significantly performed higher distance sprinting and high-speed running than those players who won the match [32]. These findings, together, could suggest that the results of a competition might have a significant impact on the players' HRV.

Nevertheless, to the best of our knowledge, no previous study has investigated the impact of the results of a soccer competition on the HRV of female soccer players. Given the preceding, the purpose of this study was to investigate if the results of competition could impact the HRV of female soccer players. Thus, a follow-up of soccer players during two weeks of the league, using an HRV recorded two days before the competition (baseline) and one recorded after the competition (post-competition), was conducted. We hypothesized that HRV would be decreased after both matches (a lost and a won match). Nevertheless, significant differences are found in a lost soccer match when compared with a won match.

\section{Materials and Methods}

\subsection{Participants}

A total of 14 players were assessed for eligibility. However, 13 female players (age $=23.76(5.32), n=13$ ) from the Spanish Second National Division soccer league team participated in this cross-sectional study (see Table 1). One participant could not complete the procedures since she was injured, so she was excluded from the statistical analyses. None of the participants manifested sickness in the match's week, and no intercurrence was registered.

Table 1. Descriptive data of participants.

\begin{tabular}{cc}
\hline Variable & Mean (SD) \\
\hline Age (years) & $23.75(5.32)$ \\
Competition experience (years) & $8.85(2.85)$ \\
Height $(\mathrm{cm})$ & $163.61(5.54)$ \\
Weight $(\mathrm{kg})$ & $58.30(7.54)$ \\
Body Mass Index $\left(\mathrm{kg} / \mathrm{m}^{2}\right)$ & $21.78(2.63)$ \\
\hline
\end{tabular}

Participants who complete the procedures had three sessions per week of $1 \mathrm{~h}$ and a half, and they had been participating in football competitions an average of 8.85 (2.85) years. All participants agreed and gave written consent to participate in the study. In addition, procedures were approved by the University of Extremadura research ethics committee (approval number: 180/2019).

\subsection{Procedure}

Standardized procedures and recommendations for assessing and reporting HRV results were followed [33]. Due to coach requirements, in terms of time limitation, we decided to conduct a short-term record ( $5 \mathrm{~min}$ ) for each HRV (baseline and post-competition). Nevertheless, five minutes of HRV is considered as the gold standard for short-term measurements [34], and it has shown excellent reliability for relevant variables such as RMMSD $($ ICC $=0.97(0.81-0.99))$ [35]. 
The participants' HRV was evaluated before (baseline) and after (post-competition) two matches, in the local dressing room with controlled temperature and humidity (22.3 (1.0) $\left.{ }^{\circ} \mathrm{C} ; 46.4(2.8) \%\right)$ at rest in a sitting position. In order to avoid distractions or interactions between players, at the time of HRV assessment, the room was calmed, all the players were at their places, and they were encouraged to remain silent (without talking).

The HRV measurements were on the same day of the week and at the same time for the two matches and training sessions. Players were familiarized with the procedures and environment. All participants underwent the same training, as well as the same precompetitive routines during the two selected microcycles. Moreover, participants did not take any drug, drink, or other substance that could affect the nervous system $24 \mathrm{~h}$ before undergoing the protocol.

The same following procedures were carried out in each match (Figure 1):

(1) Baseline: HRV data was recorded two days before the match (in order to avoid precompetition anxiety response). All participants were evaluated in the same training session before the warm-up to obtain the baseline data of the players. This data was used to normalize the data obtained in the post-competition measure.

(2) Post-competition: HRV data was recorded immediately after the match in a 5minute register.

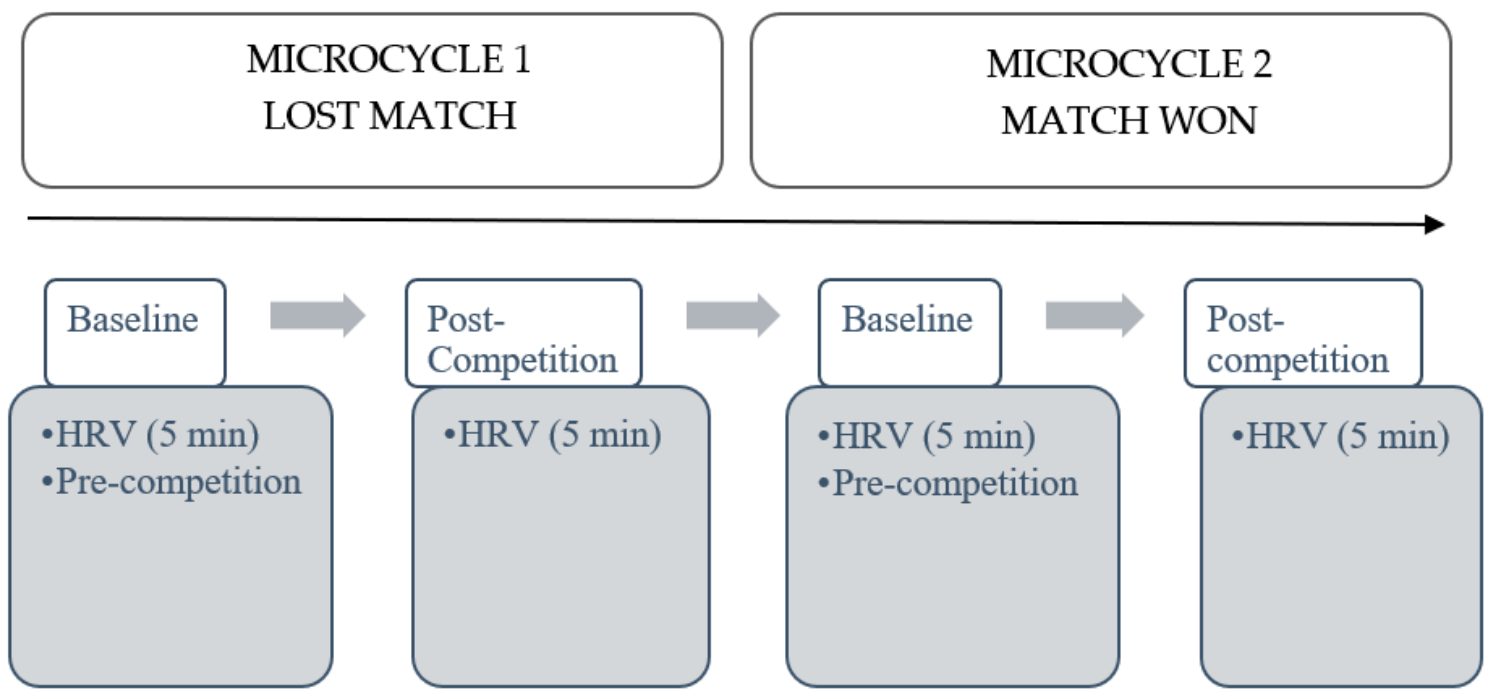

Figure 1. Study procedure timeline. HRV: Heart rate variability.

One match was lost, and the other was won, so this allowed us to evaluate if the psychophysiological impact is different between a lost or a won match.

\subsection{Instruments and Outcomes}

The HRV data was assessed using the Polar RS800CX (Polar Electro Ltd., Kempele, Finland) heart rate monitor [36] during $5 \mathrm{~min}$ at a sampling frequency of $1000 \mathrm{~Hz}$ without controlling breathing. HRV data was analyzed using the software Kubios HRV software (v. 3.3; Kubios Oy, Kuopio, Finland) [37]. A middle filter was applied to correct possible artefacts, identifying those R-to-R waves intervals shorter/longer than $0.25 \mathrm{~s}$, compared to the average of the previous beats. Correction replaces the identified artefacts with cubic spline interpolation.

Time, frequency, and nonlinear domains were analyzed: (1) time domain, such as mean heart rate (mean HR), RR intervals, RR50 count divided by the total number of all RR ranges (Pnn50), and the square root of differences between adjacent RR intervals (RMSSD); (2) frequency domain, including low-frequency (LF, 0.04-0.15 Hz) and high-frequency (HF, 0.15-0.4 Hz) ratio (LF/HF) and total power; and (3) nonlinear measures, such as RR variability from heartbeat to short-term Poincaré graph (width) (SD1) and RR variability 
from heartbeat to long-term Poincaré graph (length) (SD2). Moreover, the stress index, the parasympathetic nervous system index (PNS index), and the sympathetic nervous system index (SNS index) were calculated. The stress index is the square root of the Baevsky's stress. The PNS index was calculated based on mean RR (ms), RMSSD (ms), and SD1 $(\%)$. The SNS index was calculated based on mean HR (bpm), Baevsky's stress index, and SD2 (\%).

\subsection{Statistical Analysis}

The IBM SPSS (Statistical Package for Social Sciences, version 25; IBM, Armonk, NY, USA) statistical package was used to analyze the data.

The Shapiro-Wilk test was conducted to explore the distribution of the HRV data since the sample size was under 50 [38]. Taking into account both the results of this test (see Supplementary Table S1) and the small sample size, nonparametric analyses were conducted. Thus, Wilcoxon signed-rank tests were conducted to explore differences between baseline and post-competitive HRV measures in both the loss and the win matches.

Subsequently, HRV data was normalized for each match (calculating the difference between match and baseline data for each HRV variable). Once HRV variables were normalized, Wilcoxon signed-rank tests were performed to explore the differences between the loss and the win matches. The effect sizes ( $\mathrm{r}$ ) were calculated. It is classified as follows: 0.5 is a large effect, 0.3 is a medium effect, and 0.1 is a small effect $[39,40]$.

\section{Results}

Table 2 shows the values of the HRV at baseline and after losing a soccer match. Significant differences between baseline and post-competition HRV values were found. In this regard, HRV significantly decreased all the studied variables ( $p$-value $<0.05$ ).

Table 2. HRV at baseline and after losing a soccer match for female soccer athletes.

\begin{tabular}{ccccc}
\hline Variable & $\begin{array}{c}\text { Baseline } \\
\text { Mean (SD) }\end{array}$ & $\begin{array}{c}\text { Post-Competition } \\
\text { Mean (SD) }\end{array}$ & $p$-Value & Effect Size \\
\hline PNS Index & $0.40(1.69)$ & $-2.42(0.48)$ & $0.002^{*}$ & 0.843 \\
SNS Index & $0.55(2.98)$ & $4.45(1.84)$ & $0.013^{*}$ & 0.688 \\
Stress Index & $9.63(8.31)$ & $19.93(8.40)$ & $0.016^{*}$ & 0.669 \\
Mean HR & $72.95(20.06)$ & $104.93(10.94)$ & $0.002 *$ & 0.862 \\
RR & $860.26(154.03)$ & $578.06(65.35)$ & $0.002 *$ & 0.862 \\
pNN50 & $41.51(20.72)$ & $3.32(4.62)$ & $0.002 *$ & 0.843 \\
RMSSD & $66.84(34.23)$ & $16.72(9.51)$ & $0.002 *$ & 0.843 \\
HF & $50.17(16.76)$ & $13.06(9.29)$ & $0.002 *$ & 0.843 \\
LF & $49.74(16.80)$ & $86.90(9.32)$ & $0.002 *$ & 0.843 \\
LF/HF & $1.36(1.37)$ & $11.43(9.24)$ & $0.002 *$ & 0.843 \\
Total power & $4157.06(3956.21)$ & $682.13(711.64)$ & $0.002 *$ & 0.862 \\
SD1 & $47.34(24.25)$ & $11.83(6.73)$ & $0.002 *$ & 0.843 \\
SD2 & $73.07(33.71)$ & $34.45(16.47)$ & $0.002 *$ & 0.862 \\
\hline
\end{tabular}

* $p$-value < 0.05; HR: heart rate; RR: time between intervals R-R; pNN50: percentage of intervals > 50 ms different from the previous interval; RMSSD: the square root of the mean of the squares of the successive differences of the interval RR; LF/HF: low-frequency (LF) (ms2)/high-frequency (HF) (ms2) ratio; total power: the sum of all the spectra; PNS index: parasympathetic nervous system index, SNS index: sympathetic nervous system index and stress index; SD1: dispersion, standard deviation, of points perpendicular to the axis of line-of-identity in the Poincaré plot; SD2: dispersion, standard deviation, of points along the axis of line-of-identity in the Poincaré plot.

Table 3 shows the comparison between baseline and post-competition HRV values after winning a soccer match. Significant differences were found in PNS index, SNS index, stress index, mean HR, RR, Pnn50, RMSSD, and SD1 ( $p$-value < 0.05). 
Table 3. HRV at baseline and after winning a soccer match for female soccer athletes.

\begin{tabular}{ccccc}
\hline Variable & $\begin{array}{c}\text { Baseline } \\
\text { Mean (SD) }\end{array}$ & $\begin{array}{c}\text { Post-Competition } \\
\text { Mean (SD) }\end{array}$ & $p$-Value & Effect Size \\
\hline PNS Index & $-0.64(1.30)$ & $-1.83(0.97)$ & $0.019^{*}$ & 0.652 \\
SNS Index & $1.11(1.60)$ & $2.79(1.90)$ & $0.015^{*}$ & 0.674 \\
Stress Index & $10.73(4.23)$ & $14.49(6.03)$ & $0.041^{*}$ & 0.565 \\
Mean HR & $79.52(14.63)$ & $94.10(14.59)$ & $0.015^{*}$ & 0.566 \\
RR & $776.95(134.80)$ & $653.20(110.65)$ & $0.023^{*}$ & 0.631 \\
pNN50 & $21.36(20.10)$ & $7.67(10.73)$ & $0.023^{*}$ & 0.630 \\
RMSSD & $42.97(24.91)$ & $24.21(15.53)$ & $0.028^{*}$ & 0.609 \\
HF & $37.45(19.84)$ & $16.27(7.60)$ & $0.006^{*}$ & 0.762 \\
LF & $62.38(19.87)$ & $83.71(7.58)$ & $0.006^{*}$ & 0.762 \\
LF/HF & $2.91(3.41)$ & $6.65(4.02)$ & $0.028^{*}$ & 0.609 \\
Total power & $1956.09(1698.56)$ & $1668.89(1608.24)$ & 0.272 & 0.304 \\
SD1 & $30.43(17.65)$ & $17.14(11.00)$ & $0.028^{*}$ & 0.609 \\
SD2 & $56.28(23.42)$ & $49.25(22.56)$ & 0.182 & 0.370 \\
\hline
\end{tabular}

* $p$-value<0.005; HR: heart rate; RR: time between intervals R-R; pNN50: percentage of intervals > 50 ms different from the previous interval; RMSSD: the square root of the mean of the squares of the successive differences of the interval RR; LF/HF: low-frequency (LF) (ms2)/high-frequency (HF) (ms2) ratio; total power: the sum of all the spectra; PNS index: parasympathetic nervous system index, SNS index: sympathetic nervous system index and stress index; SD1: dispersion, standard deviation, of points perpendicular to the axis of line-of-identity in the Poincaré plot; SD2: dispersion, standard deviation, of points along the axis of line-of-identity in the Poincaré plot.

Comparisons between normalized HRV data in both the loss and the win matches are shown in Table 4. After losing a match, participants showed significant decrease in the PNS index, Pnn50, RMSSD, HF, total power, SD1, and SD2, and an increased SNS index and stress index $(p$-value $<0.05)$.

Table 4. Impact on heart rate variability of a loss and a win soccer match.

\begin{tabular}{ccccc}
\hline Variable & Loss Match & Win Match & $p$-Value & Effect Size \\
\hline \multicolumn{5}{c}{ Heart Rate Variability } \\
\hline PNS Index & $-2.83(1.64)$ & $-1.06(1.31)$ & $0.019^{*}$ & 0.549 \\
SNS Index & $3.89(3.20)$ & $1.47(1.82)$ & $0.028^{*}$ & 0.610 \\
Stress Index & $10.30(11.59)$ & $2.64(6.15)$ & $0.019^{*}$ & 0.649 \\
mean HR & $31.97(17.60)$ & $7.34(27.14)$ & $0.023^{*}$ & 0.630 \\
RR & $-282.19(137.09)$ & $-173.99(2625.27)$ & 0.116 & 0.436 \\
pNN50 & $-38.18(22.40)$ & $-14.29(20.90)$ & $0.023^{*}$ & 0.630 \\
RMSSD & $-50.12(34.73)$ & $-20.62(26.07)$ & $0.033^{*}$ & 0.591 \\
HF & $-37.11(22.28)$ & $-22.43(22.31)$ & 0.152 & 0.397 \\
LF & $37.16(22.32)$ & $14.88(25.55)$ & 0.055 & 0.533 \\
LF/HF & $10.07(9.78)$ & $3.22(5.20)$ & 0.055 & 0.533 \\
Total power & $-3474.93(3524.27)$ & $-415.57(1706.29)$ & $0.001 *$ & 0.882 \\
SD1 & $-35.50(24.60)$ & $-14.60(18.47)$ & $0.033^{*}$ & 0.591 \\
SD2 & $-38.62(24.88)$ & $-10.81(27.12)$ & $0.016^{*}$ & 0.669 \\
\hline
\end{tabular}

* $p$-value < 0.05; HR: heart rate; RR: time between intervals R-R; pNN50: percentage of intervals $>50 \mathrm{~ms}$ different from the previous interval; RMSSD: the square root of the mean of the squares of the successive differences of the interval RR; LF/HF: low-frequency (LF) (ms2)/high-frequency (HF) (ms2) ratio; total power: the sum of all the spectra; PNS index: parasympathetic nervous system index, SNS index: sympathetic nervous system index and stress index; SD1: dispersion, standard deviation, of points perpendicular to the axis of line-of-identity in the Poincaré plot; SD2: dispersion, standard deviation, of points along the axis of line-of-identity in the Poincaré plot.

\section{Discussion}

The present article aimed to assess the effects of a lost match and a won match on the post-competitive HRV in female soccer athletes. We hypothesized that HRV would be decreased after a lost soccer match compared to a won match. Results showed that HRV significantly decreased after both a lost and a won match. In this regard, comparing the impact of the two matches (a won and a lost match), a lost match induced a significant 
decrease in HRV variables (RR, pNN50, RMSSD, total power, SD1, and SD2) compared with the post-competitive HRV values obtained after the match won.

Among the tools studied to assess fatigue, HRV has emerged as a helpful tool that provides an indirect evaluation of the balance between sympathetic and parasympathetic nervous systems [34]. Thus, HRV monitoring has been used to prevent overtraining or to manage fatigue in sports such as soccer $[13,14]$ or basketball [41]. Our results showed that a lost match induced a decrease in HRV variables such as RR, pNN50, RMSSD, total power, SD1, and SD2. In this regard, previous studies have highlighted the importance of RMSSD in the identification of fatigue $[9,18]$. In the same line, Proietti, di Fronso, Pereira, Bortoli, Robazza, Nakamura and Bertollo [18] showed that RMSSD is a useful HRV variable to control the training effects in professional soccer players. Therefore, these results suggest that this variable would be quite interesting in managing fatigue in female soccer players.

The physical and mental impact of losing a soccer match could explain the results obtained. Regarding the impact of physical load in the HRV, previous studies have found that increasing physical activity intensity can significantly impact the HRV [42]. A reduced HRV after a stressor itself (a soccer match) had ceased can be due to homeostatic processes. In this regard, gluconeogenesis would presumably take place, which allows muscles and the liver to refill their energy substrates. This state would require an extra cardiac output, so a higher HR, mediated by an increase in the sympathetic modulation [43,44], can be expected. In addition, the sympathetic modulation can be significantly impacted by proinflammatory cytokines [44] induced by exercises of high duration and intensity [45]. In this line, a previous study showed that soccer players who lose a match significantly performed higher distance sprinting and high-speed running than those who won the match [32]. This could explain that players showed reduced HRV after losing a match.

Regarding the impact of mental processes on HRV, previous studies have reported that athletes experienced mood and wellbeing changes after a match loss [30,31]. Athletes reported higher depression, anxiety, social dysfunction, and anger after a loss than a win, while lower levels of vigor were observed after a loss, compared to after a win [31]. Taking into account the impact of anxiety [46] or depression [47] on the HRV, as well as the role of emotions on HRV [29], decreases in HRV variables could also be justified by this reason. Therefore, the results of a competition could be considered a relevant variable to consider when programming training load. However, HRV can be considered as an index of overall fatigue, where physical and mental states can be affected. Thus, our results should be taken with caution, since the evaluation only assessed before and after two matches, and the total volume of completed load was not taken into account. Therefore, it would be necessary to independently evaluate the total volume of completed load due to the significant impact of physical load on the HRV [48]. Future studies should independently explore the role of mental and physical components in reducing HRV after a lost match.

Results indicate that five-minute pre-and post-game HRV measurements appear to be a useful way of monitoring the state of sympathetic and parasympathetic balance in female soccer players. This is relevant, since the analysis of this monitoring would be helpful to prevent fatigue by managing training loads before matches. In this regard, previous studies showed that variables such as RMSSD or the stress index are biomarkers of internal load, and therefore are sensitive enough to detect fatigue $[49,50]$. This is extremely useful since the player cannot voluntarily alter HRV results, unlike subjective scales such as wellness or stress questionnaires [51]. However, different protocols of HRV monitoring during sports seasons can be found in the scientific literature [13-18]. In this regard, Ravé and Fortrat [13] conducted the HRV analysis while players performed a 10 min phase in the supine position followed by a $7 \mathrm{~min}$ standing phase during a 5-week training period. Thorpe, Strudwick, Buchheit, Atkinson, Drust and Gregson [15] recorded the HRV during 5 min seated after $5 \mathrm{~min}$ cycling $/ 5 \mathrm{~min}$ recovery at $130 \mathrm{~W}(85 \mathrm{rpm})$. Boullosa, Abreu, Nakamura, Muñoz, Domínguez and Leicht [14] assessed the weekly HRV, from the mean of four-daily, continuous $3 \mathrm{~h}$ nighttime recordings. However, there are similar protocols, but not identical to ours (5 min at rest). In this regard, Vilamitjana, Lentini, Pérez-Júnior 
and Verde [16] measured the HRV during 5 min immediately after awakening on the match day. Botek, Krejčí, McKune and Klimešová [17] recorded 300 artefact-free subsequent RR intervals at rest. In the same line, Proietti, di Fronso, Pereira, Bortoli, Robazza, Nakamura and Bertollo [18] recorded 10 min seated. However, any of the protocols can be measured before and after the match. Taking into account the variability in the procedures, future studies should explore the feasibility of these protocols, trying to standardize them. This would allow the comparison with other studies.

One limitation of the present study is the relatively small sample size. Nevertheless, soccer is a sport discipline of great relevance and impact on society, and women are increasing their presence in this game. However, interfering with the dynamics of individual players on a training or match day is a drawback when it comes to obtaining a larger number of women participants. We were aware that it would be difficult for volunteers to complete the study due to, for example, injuries (a player was lost for this reason) or poor individual play. For all these reasons, we considered that, given the magnitude of the championship (second division of the Spanish female soccer league), the sample to be studied and the results obtained are of enormous interest. Furthermore, the evaluation of $\mathrm{HRV}$ only in relation to the results of the competition (win or lose) is very partial, and future studies should also evaluate the total volume of completed load, which affects the spectrum HRV. Moreover, it would be interesting to analyze the impact that the player's position on the field (i.e., goalkeeper, central, midfield, or forward) can have on the HRV [52-55]. It is possible that a different impact on HRV can be observed depending on the aerobic and anaerobic requirements for each position on the field. Therefore, continuous and systematic analyses throughout the season can allow for individual monitoring of each player, providing valuable information for adjusting training loads and/or detecting possible interventions on a psychological level $[11,21,56]$. Lastly, due to time limitation and staff's requirement, HRV assessment lasted $5 \mathrm{~min}$. Although it is the gold standard for short-term measurements [34], future studies should investigate the specific reliability of this assessment in female soccer players.

\section{Conclusions}

The result of a soccer competition might significantly impact the HRV of female soccer players. In this regard, a lost match led to a decrease in HRV when compared with a match won. Therefore, researchers, coaches, or physical trainers should take into account the results of a competition when programming training load, since fatigue might be higher after a lost soccer match.

Supplementary Materials: The following are available online at https:/ /www.mdpi.com/article/10 .3390/ijerph18179414/s1, Table S1: Shapiro-Wilk test.

Author Contributions: Conceptualization, R.M.A.-M.; data curation, R.M.A.-M. and S.V.; formal analysis, S.V.; funding acquisition, H.N.; investigation, R.M.A.-M.; methodology, J.P.F.-G. and S.V.; resources, R.M.A.-M. and H.N.; software, S.V.; supervision, H.N.; validation, J.P.F.-G., H.N. and S.V.; visualization, J.P.F.-G.; writing — original draft, R.M.A.-M. and S.V.; writing—review and editing, J.P.F.-G., H.N. and S.V. All authors have read and agreed to the published version of the manuscript.

Funding: This study has been made thanks to the contribution of the Ministry of Economy and Infrastructure of the Junta de Extremadura through the European Regional Development Fund. A way to make Europe (GR18129).

Institutional Review Board Statement: The study was conducted according to the guidelines of the Declaration of Helsinki, and approved by the Ethics Committee of the University of Extremadura (protocol code: 180/2019).

Informed Consent Statement: Informed consent was obtained from all subjects involved in the study.

Data Availability Statement: Data will be available upon reasonable request to the corresponding author.

Conflicts of Interest: The authors declare no conflict of interest. 


\section{References}

1. Casamichana, D.; Castellano, J. Time-motion, heart rate, perceptual and motor behaviour demands in small-sides soccer games: Effects of pitch size. J. Sports Sci. 2010, 28, 1615-1623. [CrossRef]

2. Manso, J.M.G. Aplicación de la variabilidad de la frecuencia cardiaca al control del entrenamiento deportivo: Análisis en modo frecuencia. Arch. Med. Deporte 2013, 30, 43-51.

3. Halson, S.L. Monitoring training load to understand fatigue in athletes. Sports Med. 2014, 44, 139-147. [CrossRef]

4. Bourdon, P.C.; Cardinale, M.; Murray, A.; Gastin, P.; Kellmann, M.; Varley, M.C.; Gabbett, T.J.; Coutts, A.J.; Burgess, D.J.; Gregson, W. Monitoring athlete training loads: Consensus statement. Int. J. Sports Physiol. Perform. 2017, 12, S2161-S2170. [CrossRef] [PubMed]

5. Walker, A.J.; McFadden, B.A.; Sanders, D.J.; Rabideau, M.M.; Hofacker, M.L.; Arent, S.M. Biomarker Response to a Competitive Season in Division I Female Soccer Players. J. Strength Cond. Res. 2019, 33, 2622-2628. [CrossRef] [PubMed]

6. Thayer, J.F.; Ahs, F.; Fredrikson, M.; Sollers, J.J., III; Wager, T.D. A meta-analysis of heart rate variability and neuroimaging studies: Implications for heart rate variability as a marker of stress and health. Neurosci. Biobehav. Rev. 2012, 36, 747-756. [CrossRef] [PubMed]

7. Shaffer, F.; McCraty, R.; Zerr, C.L. A healthy heart is not a metronome: An integrative review of the heart's anatomy and heart rate variability. Front. Psychol. 2014, 5, 1040. [CrossRef]

8. Mourot, L.; Bouhaddi, M.; Perrey, S.; Cappelle, S.; Henriet, M.T.; Wolf, J.P.; Rouillon, J.D.; Regnard, J. Decrease in heart rate variability with overtraining: Assessment by the Poincare plot analysis. Clin. Physiol. Funct. Imaging 2004, 24, 10-18. [CrossRef]

9. Schmitt, L.; Regnard, J.; Millet, G.P. Monitoring fatigue status with HRV measures in elite athletes: An avenue beyond RMSSD? Front. Physiol. 2015, 6, 343. [CrossRef]

10. Rodas, G.; Yanguas, X.; Pedret, C.; Ramos, J.; Capdevila, L. Cambios en la variabilidad de la frecuencia cardiaca (VFC) en jugadores de hockey hierba durante el Campeonato del Mundo de 2006. Apunts. Med. De L'esport 2011, 46, 117-123. [CrossRef]

11. Moreno Sánchez, J.; Parrado Romero, E.; Capdevila Ortís, L. Variabilidad de la frecuencia cardíaca y perfiles psicofisiológicos en deportes de equipo de alto rendimiento. Rev. De Psicol. Del Deporte 2013, 22, 0345-0352.

12. Buchheit, M.; Racinais, S.; Bilsborough, J.C.; Bourdon, P.C.; Voss, S.C.; Hocking, J.; Cordy, J.; Mendez-Villanueva, A.; Coutts, A.J. Monitoring fitness, fatigue and running performance during a pre-season training camp in elite football players. J. Sci. Med. Sport 2013, 16, 550-555. [CrossRef]

13. Ravé, G.; Fortrat, J.-O. Heart rate variability in the standing position reflects training adaptation in professional soccer players. Eur. J. Appl. Physiol. 2016, 116, 1575-1582. [CrossRef] [PubMed]

14. Boullosa, D.A.; Abreu, L.; Nakamura, F.Y.; Muñoz, V.E.; Domínguez, E.; Leicht, A.S. Cardiac autonomic adaptations in elite Spanish soccer players during preseason. Int. J. Sports Physiol. Perform. 2013, 8, 400-409. [CrossRef] [PubMed]

15. Thorpe, R.T.; Strudwick, A.J.; Buchheit, M.; Atkinson, G.; Drust, B.; Gregson, W. Monitoring fatigue during the in-season competitive phase in elite soccer players. Int. J. Sports Physiol. Perform. 2015, 10, 958-964. [CrossRef] [PubMed]

16. Vilamitjana, J.J.; Lentini, N.A.; Pérez-Júnior, M.F.; Verde, P.E. Heart rate variability as biomarker of training load in professional soccer players. Med. Sci. Sports Exerc. 2014, 46, 1-7. [CrossRef]

17. Botek, M.; Krejčí, J.; McKune, A.J.; Klimešová, I. Somatic, endurance performance and heart rate variability profiles of professional soccer players grouped according to age. J. Hum. Kinet. 2016, 54, 65. [CrossRef]

18. Proietti, R.; di Fronso, S.; Pereira, L.A.; Bortoli, L.; Robazza, C.; Nakamura, F.Y.; Bertollo, M. Heart rate variability discriminates competitive levels in professional soccer players. J. Strength Cond. Res. 2017, 31, 1719-1725. [CrossRef]

19. Flatt, A.A.; Esco, M.R. Smartphone-derived heart-rate variability and training load in a women's soccer team. Int. J. Sports Physiol. Perform. 2015, 10, 994-1000. [CrossRef]

20. Heathers, J.A.J. Smartphone-enabled pulse rate variability: An alternative methodology for the collection of heart rate variability in psychophysiological research. Int. J. Psychophysiol. 2013, 89, 297-304. [CrossRef]

21. Ayuso-Moreno, R.; Fuentes-García, J.P.; Collado-Mateo, D.; Villafaina, S. Heart rate variability and pre-competitive anxiety according to the demanding level of the match in female soccer athletes. Physiol. Behav. 2020, 222, 112926. [CrossRef]

22. Thorpe, R.T.; Atkinson, G.; Drust, B.; Gregson, W. Monitoring fatigue status in elite team-sport athletes: Implications for practice. Int. J. Sports Physiol. Perform. 2017, 12, S227-S234. [CrossRef]

23. Marqués, D.; Calleja, J.; Arrabatiel, I.; Terrados, N. Fatiga y daño muscular en fútbol: Un proceso complejo. Rev. De Prep. Física En El Fútbol 2016, 21, 19-29.

24. Badin, O.O.; Smith, M.R.; Conte, D.; Coutts, A.J. Mental fatigue: Impairment of technical performance in small-sided soccer games. Int. J. Sports Physiol. Perform. 2016, 11, 1100-1105. [CrossRef]

25. Mohr, M.; Krustrup, P.; Bangsbo, J. Fatigue in soccer: A brief review. J. Sports Sci. 2005, 23, 593-599. [CrossRef] [PubMed]

26. Rampinini, E.; Coutts, A.J.; Castagna, C.; Sassi, R.; Impellizzeri, F.M. Variation in top level soccer match performance. Int. J. Sports Med. 2007, 28, 1018-1024. [CrossRef]

27. Fuentes-García, J.P.; Pereira, T.; Castro, M.A.; Santos, A.C.; Villafaina, S. Psychophysiological stress response of adolescent chess players during problem-solving tasks. Physiol. Behav. 2019, 209, 112609. [CrossRef] [PubMed]

28. Villafaina, S.; Collado-Mateo, D.; Cano-Plasencia, R.; Gusi, N.; Fuentes, J.P. Electroencephalographic response of chess players in decision-making processes under time pressure. Physiol. Behav. 2019, 198, 140-143. [CrossRef] 
29. Mather, M.; Thayer, J.F. How heart rate variability affects emotion regulation brain networks. Curr. Opin. Behav. Sci. 2018, 19, 98-104. [CrossRef] [PubMed]

30. Sloman, L.; Sturman, E.D.; Price, J.S. Winning and losing: An evolutionary approach to mood disorders and their therapy. Can. J. Psychiatry 2011, 56, 324-332. [CrossRef] [PubMed]

31. Jones, M.V.; Sheffield, D. The impact of game outcome on the well-being of athletes. Int. J. Sport Exerc. Psychol. 2007, 5, 54-65. [CrossRef]

32. Moalla, W.; Fessi, M.S.; Makni, E.; Dellal, A.; Filetti, C.; Di Salvo, V.; Chamari, K. Association of physical and technical activities with partial match status in a soccer professional team. J. Strength Cond. Res. 2018, 32, 1708-1714. [CrossRef] [PubMed]

33. Catai, A.M.; Pastre, C.M.; de Godoy, M.F.; da Silva, E.; de Medeiros Takahashi, A.C.; Vanderlei, L.C.M. Heart rate variability: Are you using it properly? Standardisation checklist of procedures. Braz. J. Phys. Ther. 2020, 24, 91-102. [CrossRef]

34. Shaffer, F.; Ginsberg, J.P. An overview of heart rate variability metrics and norms. Front. Public Health 2017, 5, 258. [CrossRef]

35. Hoffmann, B.; Flatt, A.A.; Silva, L.E.V.; Młyńczak, M.; Baranowski, R.; Dziedzic, E.; Werner, B.; Gąsior, J.S. A Pilot Study of the Reliability and Agreement of Heart Rate, Respiratory Rate and Short-Term Heart Rate Variability in Elite Modern Pentathlon Athletes. Diagnostics 2020, 10, 833. [CrossRef]

36. de Rezende Barbosa, M.P.C.; Silva, N.T.d.; de Azevedo, F.M.; Pastre, C.M.; Vanderlei, L.C.M. Comparison of P olar ${ }^{\circledR}$ RS 800 G3 ${ }^{\text {TM }}$ heart rate monitor with $\mathrm{P}$ olar ${ }^{\circledR} \mathrm{S} 810 \mathrm{i}^{\mathrm{TM}}$ and electrocardiogram to obtain the series of RR intervals and analysis of heart rate variability at rest. Clin. Physiol. Funct. Imaging 2016, 36, 112-117. [CrossRef]

37. Tarvainen, M.P.; Niskanen, J.-P.; Lipponen, J.A.; Ranta-Aho, P.O.; Karjalainen, P.A. Kubios HRV-heart rate variability analysis software. Comput. Methods Programs Biomed. 2014, 113, 210-220. [CrossRef]

38. Yap, B.W.; Sim, C.H. Comparisons of various types of normality tests. J. Stat. Comput. Simul. 2011, 81, 2141-2155. [CrossRef]

39. Fritz, C.O.; Morris, P.E.; Richler, J.J. Effect Size Estimates: Current Use, Calculations, and Interpretation. J. Exp. Psychol. -Gen. 2012, 141, 2-18. [CrossRef]

40. Coolican, H. Research Methods and Statistics in Psychology; Psychology Press: Hove, UK, 2017.

41. Mazon, J.H.; Gastaldi, A.C.; Martins-Pinge, M.C.; Eduardo de Araújo, J.; Dutra de Souza, H.C. Study of Heart Rate Variability and Stress Markers in Basketball Players Submitted to Selective Loads Periodization System. Am. J. Sports Sci. 2015, 3, 46-51. [CrossRef]

42. Hunt, K.J.; Saengsuwan, J. Changes in heart rate variability with respect to exercise intensity and time during treadmill running. Biomed. Eng. Online 2018, 17, 1-12. [CrossRef]

43. Gerich, J.E. Control of glycaemia. Bailliere's Clin. Endocrinol. Metab. 1993, 7, 551-586. [CrossRef]

44. Kimura, K.; Ieda, M.; Fukuda, K. Development, maturation, and transdifferentiation of cardiac sympathetic nerves. Circ. Res. 2012, 110, 325-336. [CrossRef]

45. Bigard, A.X. Lésions musculaires induites par l'exercice et surentraînement. Sci. Sports 2001, 16, 204-215. [CrossRef]

46. Chalmers, J.A.; Quintana, D.S.; Abbott, M.J.; Kemp, A.H.J.F.i.p. Anxiety disorders are associated with reduced heart rate variability: A meta-analysis. Front. Psychiatry 2014, 5, 80. [CrossRef]

47. Hartmann, R.; Schmidt, F.M.; Sander, C.; Hegerl, U. Heart rate variability as indicator of clinical state in depression. Front. Psychiatry 2019, 9, 735. [CrossRef]

48. Kaikkonen, P.; Hynynen, E.; Mann, T.; Rusko, H.; Nummela, A. Heart rate variability is related to training load variables in interval running exercises. Eur. J. Appl Physiol. 2012, 112, 829-838. [CrossRef]

49. Jimenez, C.N.; Álvarez, J.R.; Mainer, E.P.; Orellana, J.N. La variabilidad de la frecuencia cardiaca en el control del entrenamiento en un corredor de Ironman: Estudio de caso. Retos: Nuevas Tend. En Educ. Físicadeporte Y Recreación 2020, 37, $339-343$.

50. Nieto-Jiménez, C.; Pardos-Mainer, E.; Ruso-Álvarez, J.F.; Naranjo-Orellana, J. Training Load and HRV in a Female Athlete: A Case Study. Rev. Int. De Med. Y Cienc. De La Act. Física Y El Deporte 2020, 20, 321-333.

51. Forner-Llacer, F.; Aranda-Malaves, R.; Malavés, R.A.; Calleja-Gonzalez, J.; Perez-Turpin, J.A.; Gonzalez-Rodenas, J. Minimal stabilization time for ultra-short heart rate variability measurements in professional soccer. Int. J. Sports Med. 2020, 41, 1032-1038.

52. Muñiz-González, J.; Giráldez-Costas, V.; González-García, J.; Romero-Moraleda, B.; Campos-Vázquez, M.Á. Diferencias posicionales en las fases de máxima exigencia condicional en fútbol femenino. [Positional differences in the most demanding conditional phases in female football competition]. RICYDE Revista Internacional de Ciencias del Deporte 2020, 16, 199-213. [CrossRef]

53. Reilly, T. Energetics of high-intensity exercise (soccer) with particular reference to fatigue. J. Sports Sci. 1997, 15, 257-263. [CrossRef] [PubMed]

54. Rodriguez-Alonso, M.; Fernandez-Garcia, B.; Perez-Landaluce, J.; Terrados, N. Blood lactate and heart rate during national and international women's basketball. J. Sports Med. Phys. Fit. 2003, 43, 432.

55. Alvarez Torrella, S. Marcadores de Fatiga en Fútbol. 2016. Available online: http://dspace.umh.es/bitstream/11000/2847/1 /TFG\%20Alvarez\%20Torrella\%2C\%20Sergio.pdf (accessed on 4 September 2021).

56. Abbott, W.; Brownlee, T.E.; Harper, L.D.; Naughton, R.J.; Clifford, T. The independent effects of match location, match result and the quality of opposition on subjective wellbeing in under 23 soccer players: A case study. Res. Sports Med. 2018, 26, 262-275. [CrossRef] 